P801

\section{MULTICOMPONENT PROGRAM OF MEDICAL REHABILITATION OF PATIENTS WITH \\ OSTEOARTHRITIS AND RHEUMATOID ARTHRITIS}

\section{N. V. Aleksandrova ${ }^{1}$, A. V. Aleksandrov ${ }^{1}$}

${ }^{1}$ Federal State Budgetary Institution, Research Institute of Clinical and Experimental Rheumatology A.B. Zborovsky, Volgograd, Russia

Objective: To evaluate the efficiency of the developed multicomponent rehabilitation program in patients with osteoarthritis (OA) and rheumatoid arthritis (RA) during sanatorium-and-spa treatment.

Methods: We observed 60 patients with OA (48 women and 12 men, the average age was 57.4 [49.3: 71.1] years old, the median duration of the disease was 14.8 [5.5: 21.9] y), and 34 patients with RA ( 30 women and 4 men, average age - 45.2 [37.9: 60.2] years old, median disease duration - 9.6 [3.9: 14.1] years old, with low activity of the pathological process (DAS28 $<3.2$ ). The following rehabilitation technologies (RT) were used at the sanatorium-resort stage of medical rehabilitation (within $21 \mathrm{~d}$ ): I - kinesitherapy (dosed walking from 30-60 min daily), II - hydrokinesitherapy (therapeutic swimming in a pool with seawater, 10-12 procedures for 30 min daily or every other day), III - low-frequency magnetotherapy (from $0.3-100 \mathrm{~Hz}$; up to 5 $\mathrm{mT} ; 10$ procedures for 30 min, every other day), IV - a method of functional biocontrol with biofeedback (BFB therapy) (10-12 sessions of thermal and myographic BFB therapy, for 20-25 min daily). The intensity of pain in the affected joints (when walking) was assessed using a visual analogue scale (VAS Huskisson), swelling and pain on palpation of the joints (in absolute numbers), ESR, CRP, and general antioxidant status (ELISA test; Immundiagnostik, Germany).

Results: The combined use of RT-I and RT-II in patients with OA $(n=24)$ reduced the intensity of pain $(p=0.041)$ and the number of painful joints $(p=0.048)$, and in patients with $R A(n=10)$ reduced pain on the VAS scale $(p=0.029)$. The combined use of RT-III and RT-IV in patients with $O A(n=20)$ reduced the intensity of pain $(p=0.017)$ and the number of swollen joints $(p=0.044)$, and it reduced ESR ( $p=0.033)$ and increased the indicators of the general antioxidant status $(p=0.019)$ in patients with RA $(n=10)$. The use of multicomponent treatment regimens (a combination of all RTs) showed a significant decrease in pain intensity $(p=0.022)$, the number of painful and swollen joints $(p=0.034$ and $p=0.049)$, ESR levels $(p=0.042)$ and CRP $(p=0.01)$ in the group of patients with $O A(n=16)$. It also led to a pain relief on the VAS scale $(p=0.023)$, decrease in the number of painful joints $(p=0.031)$, the level of ESR $(p=0.018), \operatorname{CRP}(P=0.038)$ and increase in total antioxidant status indicators $(p=0.007)$ in the group of RA patients $(n=10)$.

Conclusion: The dynamics of the articular syndrome index and immuno-inflammatory complex indicate a positive result of the use of a multicomponent treatment regimen (based on modern physiotherapy methods) at the stage of medical rehabilitation of patients with joint pathology.
P802

\section{A MODIFIED TAPE TRANSFER APPROACH FOR RAPIDLY PREPARING HIGH-QUALITY CRYOSECTIONS OF UNDECALCIFIED ADULT BONES}

\author{
L. W. Zhang ${ }^{1}$, Y. J. Yang ${ }^{2}$, M. Zhu ${ }^{3}$, X. J. Fu' ${ }^{2}$, J. Q. Chen², D. \\ Hong ${ }^{1}$ \\ 'Orthopaedic Department, Taizhou Hospital of Zhejiang Province, \\ Wenzhou Medical University; Orthopedic Institute, Soochow \\ University, Linhai, ${ }^{2}$ Orthopedic Institute, Soochow University, \\ Soochow, ${ }^{3}$ Orthopaedic Department, Taizhou Hospital of \\ Zhejiang Province, Wenzhou Medical University, Linhai, China
}

Objective: Histology-based analyses are important tools to dissect cellular and molecular mechanisms of skeletal homeostasis, diseases, and regeneration. The success of these efforts is highly dependent on rapidly obtaining high-quality sections of mineralized skeletal tissues suitable for various analyses. However, the current techniques for preparing such sections are still far from satisfactory.

Methods: Here, we reported the development of a modified version of tape transfer approach that can prepare cryosections of undecalcified rodent adult bones within $4 \mathrm{~d}$ at a low cost. This approach involved two important modifications to Cryojane TapeTransfer System, including utilization of an optimized adhesive to prepare adhesive glass slides for improving the transfer efficiency, and a cheap conventional benchtop UV transilluminator for UV curing.

Results: Bone sections prepared by this approach exhibited good tissue morphology and structural integrity. Moreover, these sections were applicable to a variety of histological analyses, including calcein labeling, von Kossa staining, immunofluorescence, and enzymatic activity staining as well as EdU and TUNEL assays. Compared with traditional plastic sectioning that is widely used for preparing sections of mineralized tissues, our approach holds the following advantages. Firstly, we can rapidly obtain the specimen sections usually within $4 \mathrm{~d}$, including one day of sample fixation, and two days of sample cryoprotection and embedding, before samples are sectioned on the fourth day. Secondly, our method has a relatively lower cost than traditional plastic sectioning, since we utilize custom-made adhesive slides (about $\$ 0.2 /$ slide), regular disposable blades, and a cheap benchtop UV transilluminator instead of expensive Cryojane Tape-Transfer system. Thirdly, cryosectioning nature of our approach better suits preservation of antigenicity and enzymatic activity.

Conclusion: Thus, the tape transfer approach we developed provides a rapid, affordable, and easy learning method for preparing high-quality undecalcified bone sections valuable for bone research.

Acknowledgments: This work is supported by the National Natural Science Foundation of China (81974344, 81772294), the Natural Science Foundation of Huai'an (HAB201848), the Zhejiang 